\title{
Activity Assessment of Eosinophilic Esophagitis
}

\author{
Alain Schoepfer ${ }^{a}$ Ekaterina Safroneeva ${ }^{b}$ \\ a Division of Gastroenterology and Hepatology, Centre Hospitalier Universitaire Vaudois/CHUV, Lausanne, and \\ ${ }^{\mathrm{b}}$ Institute for Social and Preventive Medicine, University of Bern, Bern, Switzerland
}

\begin{abstract}
Key Words
Eosinophilic esophagitis · Patient-reported outcomes ·

Biologic measures
\end{abstract}

\begin{abstract}
The activity of eosinophilic esophagitis (EoE) can be assessed with patient-reported outcomes and biologic measures. Patient-reported outcomes include symptoms and quality of life, whereas biologic measures refer to endoscopic, histologic, and biochemical activity (e.g. blood biomarkers). So far, a validated tool to assess EoE activity in the above-mentioned dimensions is lacking. Given the lack of a standardized way to assess EoE activity in the various dimensions, the results of different clinical trials may be difficult to compare. For symptom assessment in adult patients, the symptom 'dysphagia' should be evaluated according to different standardized food consistencies. Furthermore, symptom assessment should take into account the following items: avoidance of specific food categories, food modification, and time to eat a regular meal. A distinct symptom recall period (e.g. 2 weeks) has to be defined for symptom assessment. Performing an 'esophageal stress test' with ingestion of a standardized meal to measure symptom severity bears the potential risk of acute food bolus impaction and should therefore be avoided. The description of endoscopic findings in EoE has meanwhile been standardized. Histologic evaluation of EoE activity should report either the size of the high-power field used or count the eosinophils
\end{abstract}

per $\mathrm{mm}^{2}$. There is a current lack of blood biomarkers demonstrating a good correlation with histologic activity in esophageal biopsies. The development and validation of an adult and pediatric EoE activity index is urgently needed not only for clinical trials and observational studies, but also for daily practice.

(c) 2014 S. Karger AG, Basel

\section{Defining Disease Activity of Eosinophilic Esophagitis: General Considerations}

Eosinophilic esophagitis (EoE) has recently been defined by a panel of international experts as 'a chronic, immune/antigen-mediated, esophageal disease characterized clinically by symptoms related to esophageal dysfunction and histologically by eosinophil-predominant inflammation' [1]. Notably, other diseases associated with esophageal eosinophil-predominant inflammation, such as achalasia or esophageal Crohn's disease, need to be ruled out before EoE can be diagnosed [2]. The definition of EoE incorporates two diagnostic pillars: symptoms and histology. This duality in diagnosis suggests that there is no 'general' EoE activity to be measured, but rather that EoE activity should be assessed at least on a symptomatic and histologic level. In addition, EoE activity can also be measured on an endoscopic level or by blood biomarkers. Figure 1 shows the different dimensions in which EoE activity can be assessed.

\section{KARGER}

E-Mail karger@karger.com

www.karger.com/ddi
(C) 2014 S. Karger AG, Basel

0257-2753/14/0322-0098\$39.50/0 
A validated activity index to assess EoE activity on different levels is currently missing. The use of different, mostly unvalidated tools to assess adult and pediatric symptom severity in EoE patients may explain why several clinical trials have documented a dissociation between EoE symptom severity and histologic activity, as measured by esophageal eosinophil counts [3, 4]. Stimulated also by the notion that $\mathrm{EoE}$ is becoming increasingly frequent with current prevalence rates of around 1 patient in 2,000 inhabitants [5, 6], we have become aware that an EoE activity index is urgently needed to define endpoints not only for clinical trials and observational studies, but also for daily clinical practice.

\section{Measuring EoE Activity: Patient-Reported Outcomes, Biologic Items, or Both?}

There is an ongoing debate as to whether EoE activity should be assessed based on patient-reported outcomes (PRO), biologic items (such as histology, endoscopy, or biomarkers), or a combination of both. The two diagnostic pillars of EoE would suggest that EoE activity should be measured at least on the level of PRO and histology. But how should the PRO and biologic markers be weighted? We think that the choice of the adequate dimensions in which a specific disease activity should be assessed strongly depends on the impact the disease's natural history has on the PRO and biologic markers. Figure 2 illustrates this appraisal with several examples.

There are diseases in which activity assessment is based mainly on PRO, such as migraine (PRO = headache; biologic marker $=$ abnormalities in functional MRI) and lower back pain $(\mathrm{PRO}=$ pain; biologic marker $=\mathrm{MRI}$ find ings). On the other end of the spectrum, there are diseases characterized by a long period during which affected patients remain asymptomatic; however, good biomarkers exist for activity assessment that are associated with hard clinical outcomes such as myocardial infarction in arterial hypertension (e.g. PRO = quality of life; biologic marker $=$ blood pressure) or hyperlipidemia (e.g. $\mathrm{PRO}=$ quality of life; biologic marker = blood lipids). In between there are diseases such as asthma (e.g. PRO = quality of life; biologic marker = FEV1) or inflammatory bowel diseases (e.g. $\mathrm{PRO}=$ bowel frequency, abdominal pain; biologic marker $=$ severity of mucosal inflammation as measured by endoscopy). As we have mentioned above, the impact of a specific disease's natural history on PRO and biologic markers determines which categories should be chosen to assess disease activity. In 2003, Straumann et al.

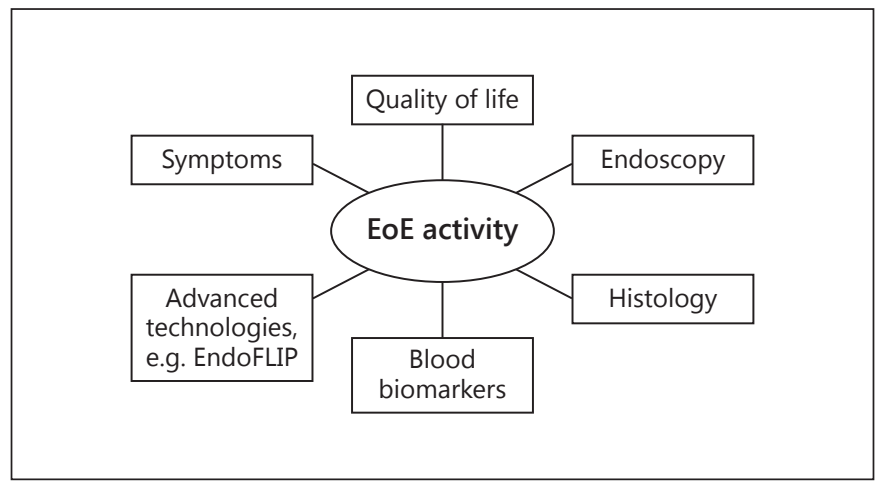

Fig. 1. Dimensions in which EoE activity can be assessed.

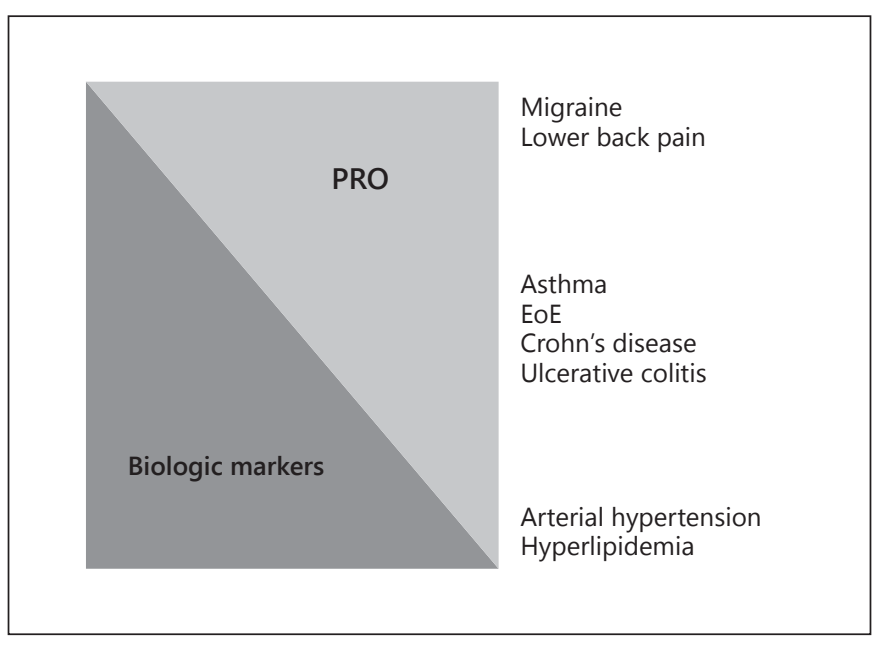

Fig. 2. PRO and biologic markers to assess activity of several diseases.

[7] were the first to report on the natural history of 30 adult EoE patients, and demonstrated that eosinophilpredominant esophageal inflammation as well as its symptoms persisted over time. A recent publication highlighted that untreated EoE leads to the formation of strictures (defined as an esophageal caliper of $10 \mathrm{~mm}$ and less) in a time-dependent manner and that endoscopic fibrotic features (rings, strictures) are evolving on top of the chronically persisting endoscopic signs of acute inflammation (whitish exudates, furrows, edema) [8]. These findings are in line with studies that have demonstrated the esophagus of untreated EoE patients undergoes remodeling processes by the deposition of subepithelial fibrous tissue $[9,10]$. All of these observations support the appraisal that EoE activity assessment should be performed using a combination of PRO and biologic markers. 


\section{Collaboration with Regulatory Authorities to Develop Clinically Meaningful Endpoints}

There is currently no Food and Drug Administration (FDA)-approved qualified symptom assessment tool for EoE, and also not for pharmacologic therapy. However, there are several ongoing clinical trials that are evaluating drugs in EoE patients. The FDA has therefore a profound interest in the identification of clinically meaningful endpoints [11]. A taskforce of the FDA has launched an initiative for a collaboration between different stakeholders dealing with EoE [11]. In their recent publication Fiorentino et al. [11] present an evolving partnership model for rational drug development in EoE. The authors also discuss the lack of correlation between PRO and histology reported by some trials $[3,4]$. In the editorial to the aforementioned article, Rothenberg et al. [12] emphasize that there has been rapid and significant progress in this relatively new disease over the last 20 years, especially regarding molecular understanding. The authors provide several persuasive explanations for the lack of correlation between PRO and histology that has been reported in several trials $[3,4]$. Furthermore, the authors raise caution that we should not become solely focused on PRO tools for regulatory product approval. The FDA is also active in the publication of guidelines regarding the development of PRO [13-15]. The concerted efforts between different stakeholders, which include patients, families, advocacy organizations, regulatory authorities, researchers, and the pharmaceutical industry, should facilitate the development of clinically meaningful endpoints to be used in clinical trials, observational studies, and daily practice.

\section{Overview of the Current Status Regarding the Development of PRO Instruments}

The development and validation of a PRO instrument to assess symptom severity in pediatric and adult EoE patients represents a challenging endeavor. The first point to consider is that symptom presentation changes with age $[1,2$, 16]. Feeding difficulties and failure to thrive are typical EoE symptoms for infants and toddlers, while school-aged children are prone to vomiting and abdominal pain $[1,2,16]$. Adolescents and adults typically present with dysphagia and food bolus impactions $[1,2,16]$. For pediatric patients, a cutoff has to be chosen from which children are able to report on symptoms themselves and up to which age a parent-reported symptom assessment should be performed. For adolescent and adult patients, dysphagia characteristics (severity, frequency) strongly depend on eating habits and should therefore be assessed using standardized food consistencies. In addition, symptom severity may depend on food avoidance, food modification, or also the time to ingest a standardized meal. Furthermore, a distinct symptom recall period (e.g. 2 weeks) should be chosen for symptom assessment. All of these factors should be taken into account when developing a PRO instrument for pediatric and adult EoE patients. Additionally, it might seem tempting at first to perform an 'esophageal stress test' with ingestion of a standardized meal to measure symptom severity; however, such a procedure bears the potential risk of acute food bolus impaction and should therefore be avoided.

What is the current status of development and validation for PRO instruments incorporating symptoms and quality of life for pediatric and adult EoE patients? In 2011, Franciosi et al. [17] reported on the qualitative methods of the Pediatric Eosinophilic Esophagitis Symptom Score (PEESS, version 2.0). The same group also recently published a report on a quality of life instrument for pediatric EoE patients [18]. For adult EoE patients, the development and validation of the Eosinophilic Esophagitis Activity Index (EEsAI) is currently ongoing (clinicaltrials.gov, NCT00939263). In addition, a quality of life questionnaire for adult EoE patients has recently been published by Hirano and coworkers [19].

\section{Overview of the Current Status Regarding the Development of Biologic Measures}

Hirano et al. [20] recently published a classification and grading system for endoscopic assessment of esophageal EoE features. However, there are currently no definitions regarding endoscopic remission and mild, moderate, or severe endoscopic activity.

The assessment of the histologic activity mainly relies on the peak eosinophil count per high-power field. Additional histologic findings such as basal layer hyperplasia, papillary elongation, eosinophil degranulation, and subepithelial fibrosis have also been reported as histologic outcome parameters. In 2007, Dellon et al. [21] showed that the number of reported peak eosinophil counts may not be necessarily comparable as different microscope types with specific highpower field sizes may be used. Reporting the observed peak eosinophil counts standardized to $\mathrm{mm}^{2}$ could overcome this issue. Like the reporting of endoscopic severity, currently there are also no definitions regarding histologic remission and different semiquantitative degrees of histologic activity.

Furuta et al. [22] recently evaluated the esophageal string test as a minimally invasive tool to assess the cor- 
relation between esophageal eosinophil counts and eosinophil granule proteins attached to the string. They found that the level of eosinophil granule proteins extracted from the string correlated well with the esophageal tissue eosinophilia. The string test may be of particular interest as a follow-up tool for pediatric EoE patients in which the threshold to perform an endoscopy is usually higher compared to the adult EoE population.

Uncontrolled eosinophil-predominant esophageal inflammation leads to remodeling with formation of strictures in the long term [8]. These structural esophageal abnormalities may be detected by the EndoFLIP tool, an inflatable bag introduced to the esophagus that measures compliance and luminal diameters [23].

\section{Outlook}

The first reports of EoE patients were published 20 years ago. Considerable progress in this relatively short time interval has been made regarding controlled clini- cal trials, understanding EoE's natural history, and endoscopic, histologic, and molecular characterization. The disease activity of EoE can be assessed in a modular approach that separates PRO from biologic items. A concerted collaboration between patients, families, regulatory authorities, clinicians, researchers, and the pharmaceutical industry is mandatory to provide the community with clinically meaningful outcomes to be assessed in clinical trials, observational studies, and daily clinical practice

\section{Acknowledgements}

This work was supported by a grant from the Swiss National Science Foundation (grant No. 32003B_135665/1).

\section{Disclosure Statement}

None.

\section{References}

1 Liacouras CA, Furuta GT, Hirano I, et al: Eosinophilic esophagitis: updated consensus recommendations for children and adults. J Allergy Clin Immunol 2011;128:3-20.

- 2 Furuta GT, Liacouras CA, Collins MH, et al: Eosinophilic esophagitis in children and adults: a systematic review and consensus recommendations for diagnosis and treatment. Gastroenterology 2007;133:1342-1363.

3 Alexander JA, Jung KW, Arora AS, et al: Swallowed fluticasone improves histologic but not symptomatic response of adults with eosinophilic esophagitis. Clin Gastroenterol Hepatol 2012;10:742-749.

-4 Pentiuk S, Putnam PE, Collins MH, et al: Dissociation between symptoms and histological severity in pediatric eosinophilic esophagitis. J Pediatr Gastroenterol Nutr 2009;48:152-160.

$\checkmark 5$ Hruz P, Straumann A, Bussmann C, et al: Escalating incidence of eosinophilic esophagitis: a 20-year prospective, population-based study in Olten County, Switzerland. J Allergy Clin Immunol 2011;128:1349-1350.

-6 Prasad GA, Alexander JA, Schleck CD, et al: Epidemiology of eosinophilic esophagitis over three decades in Olmsted County, Minnesota. Clin Gastroenterol Hepatol 2009; 7:1055-1061.

7 Straumann A, Spichtin HP, Grize L, et al: Natural history of primary eosinophilic esophagitis: a follow-up of 30 adult patients for up to 11.5 years. Gastroenterology 2003;125:1660-1669.

8 Schoepfer AM, Safroneeva E, Bussmann C, et al: Delay in diagnosis of eosinophilic esophagitis increases risk for stricture formation, in a time-dependent manner. Gastroenterology 2013;145:1230-1236.
9 Mishra A, Wang M, Pemmaraju VR, et al: Esophageal remodeling develops as a consequence of tissue specific IL-5 induced eosinophilia. Gastroenterology 2008;134:204-214

10 Kagalwalla AF, Akhtar N, Woodruff SA, et al: Eosinophilic esophagitis: epithelial mesenchymal transition contributes to esophageal remodeling and reverses with treatment. J Allergy Clin Immunol 2012;129:1387-1396.

11 Fiorentino R, Liu G, Pariser A, et al: Crosssector sponsorship of research in eosinophilic esophagitis: a collaborative model for rational drug development in rare diseases. J Allergy Clin Immunol 2012;130:613-616.

12 Rothenberg ME, Aceves S, Bonis PA, et al: Working with the US Food and Drug Administration: progress and timelines in understanding and treating patients with eosinophilic esophagitis. J Allergy Clin Immunol 2012;130:617-619.

13 Patrick DL, Burke LB, Powers JH, et al: Patient-reported outcomes to support medical product labeling claims: FDA Perspective. Value Health 2007;10(suppl 2):S125-S137.

14 Bottomley A, Jones D, Claassens L: Patientreported outcomes: assessment and current perspectives of the guidelines of the Food and Drug Administration and the reflection paper of the European Medicines Agency. Eur J Cancer 2009;45:347-353.

15 McLeod LD, Coon CD, Martin SA, et al: Interpreting patient-reported outcome results: US FDA guidance and emerging methods. Expert Rev Pharmacoecon Outcomes Res 2011;11:163-169.
16 Noel RJ, Putnam PE, Rothenberg ME: Eosinophilic esophagitis. N Engl J Med 2004;351: 940-941.

17 Franciosi JP, Hommel KA, DeBrosse CW, et al: Development of a validated patient-reported symptom metric for pediatric eosinophilic esophagitis: qualitative methods. BMC Gastroenterol 2011;11:126.

18 Franciosi JP, Hommel KA, Bendo CB, et al: PedsQL eosinophilic esophagitis module: feasibility, reliability, and validity. J Pediatr Gastroenterol Nutr 2013;57:57-66.

19 Taft TH, Kern E, Kwiatek MA, Hirano I, et al: The adult eosinophilic esophagitis quality of life questionnaire: a new measure of healthrelated quality of life. Aliment Pharmacol Ther 2011;34:790-798.

20 Hirano I, Moy N, Heckman MG, et al: Endoscopic assessment of the oesophageal features of eosinophilic oesophagitis: validation of a novel classification and grading system. Gut 2013;62:489-495.

21 Dellon ES, Aderoju A, Woosley JT, et al: Variability in diagnostic criteria for eosinophilic esophagitis: a systematic review. Am J Gastroenterol 2007;102:2300-2313.

22 Furuta GT, Kagalwalla AF, Lee JJ, et al: The oesophageal string test: a novel, minimally invasive method measures mucosal inflammation in eosinophilic oesophagitis. Gut 2013; 62:1395-1405.

23 Kwiatek MA, Hirano I, Kahrilas PJ, et al: Mechanical properties of the esophagus in eosinophilic esophagitis. Gastroenterology 2011; 140:82-90. 